\section{S1PR2's plaque attack}

\section{By Lauren Martz, Staff Writer}

Japanese researchers have shown that inhibition of sphingosine-1phosphate receptor 2 affects multiple cell types that contribute to atherosclerosis. ${ }^{1}$ The question is how to develop an antagonist suitable for chronic use, given that S1PR2 is expressed on multiple cell types.

Atherosclerosis results from chronic vascular inflammation involving macrophages, epithelial cells and smooth muscle cells in blood vessels. The vascular cells' release of inflammatory cytokines, uptake of low-density lipoprotein (LDL) cholesterol and inhibition of cholesterol efflux all contribute to the accumulation of foam that forms atherosclerotic plaques and eventually can cause blood clots. ${ }^{2}$

Although statins are the gold standard for lowering cholesterol, they do not address all of the contributing factors to plaque development-some patients on statins still have heart attacks.

"The identification of new treatments for atherosclerosis has been very dry for a long time. We have statins, but they basically lower LDL and reduce risk of heart attack," said Timothy Hla, professor of pathology and laboratory medicine and director of the Center for Vascular Biology at Weill Cornell Medical College.

In a 2007 paper published in the Arteriosclerosis, Thrombosis and Vascular Biology, researchers from Kanazawa University School of Medicine found that the sphingosine-1-phosphate receptor 1 (S1PR1; S1P1; EDG1) and S1PR3 (S1P3; EDG3) agonist FTY720 might have an antiatherosclerotic effect. ${ }^{3}$ However, the role of S1PR2 (S1P2; EDG5) in the disease has been unclear.

FTY720 from Novartis AG is marketed as Gilenya fingolimod to treat multiple sclerosis (MS).

Now, a team led by Yoh Takuwa has found that S1PR2 has the opposite effect of S1PR1 and S1PR3 on the vasculature. The group showed that inhibiting S1PR2 on vascular macrophages, endothelial cells and smooth muscle cells decreases the inflammatory response and prevents atherosclerosis.

Takuwa is professor and chair of the Department of Physiology at the Kanazawa University Graduate School of Medical Science.

The group started by crossing apolipoprotein E (ApoE)-deficient mice, which develop atherosclerosis more easily than wild-type mice, with S1pr2-deficient mice. The majority of S1pr2-/- mice died near the time of weaning due to seizures, but those that survived were studied for the development of atherosclerosis.

After four months on a high-cholesterol diet, double knockout mice had a $45 \%-55 \%$ decrease in plaque area in the abdominal aorta compared with $S 1 p r 2^{+/+} / A p o E^{-/-}$mice.

In aortas from the double knockout mice on high-cholesterol diets, mRNA expression of proinflammatory cytokines was lower and expression of anti-inflammatory IL-10 was higher than those in $S 1 \mathrm{pr}^{+/+} /$ $A p o E^{-/-}$mice. In macrophages and endothelial cells from the $S 1 p r 2^{-1-/}$ $A p o E^{-/-}$mice, the rhodopsin (Rho; Opn2) pathway also was inhibited compared with that in $S 1 p r 2^{+/+} / A p o E^{-/-}$mice.

In bone marrow-derived macrophages from the double knockouts, LDL uptake was lower and cholesterol efflux was higher than those in $S 1 p r 2^{+/+} / A p o E^{-/-}$macrophages. When macrophages from the double knockout mice were transplanted into $S 1 p r 2^{+/+} / A p o E^{-/-}$mice, total plaque area in the aorta was decreased compared with that in animals given bone marrow-derived macrophages from S1pr2 $2^{+/+} / A p o E^{-/-}$mice.

Smooth muscle cell proliferation and density, which are activities that can stabilize plaques and help reduce the risk of cardiovascular events, were increased in aortic explants from the double knockouts.

Together, the findings suggest that S1PR2's inflammatory activity in macrophages, endothelial cells and smooth muscle cells contributes to the development of atherosclerosis.

The team then showed that pharmacological inhibition of S1PR2 replicated the effects of genetic knockout. In $S 1 p r 2^{+/+} / A p o E^{-/-}$mice, the selective S1PR2 antagonist JTE-K1 decreased plaque size by about $60 \%$ compared with vehicle control.

Data were published in The Journal of Clinical Investigation. The group also included researchers from The University of Tokyo Graduate School of Medicine, Tokyo University of Agriculture \& Technology and Teikyo University School of Medicine.

\section{Novel antagonists}

According to Hla, the compound used by the Japanese team originally was developed by Japan Tobacco Inc., and the current development status of the compound is unknown. The company did not return interview requests.

Last month, Hla and colleagues at Weill Cornell also published work supporting the use of S1PR2 antagonists to prevent atherosclerosis. ${ }^{4}$ His group used a different S1PR2 antagonist from the one used by the Japanese team.

The Japanese team's next step would be to identify an S1PR2 antagonist suitable for clinical use. 
"More effective and more selective derivatives of the S1PR2 antagonist JTE-K1 should be sought systematically," Takuwa said. He added that once obtained, "those chemical compounds need to be examined for possible toxicity effects, and maximal endurable doses need to be determined in animals."

However even a compound that is highly selective for S1PR2 may not be safe. Markus van der Giet, head of the Renal Transplant Program at the Campus Benjamin Franklin of Charité-University Hospital Berlin, said vascular selectivity is the key issue because "the problem of S1PR2 signaling is the general widespread expression of the receptor everywhere in the body."

For example, he said, "We know S1PR2 is essential for auditory and vestibular function."

On the plus side, van der Giet noted that inhibition of S1PR2 appears to decrease vascular tone, which increases regional blood flow and could have a beneficial effect on blood pressure.

Hla agreed that this additional receptor selectivity may be important. According to him, the Japanese team "needs to search for a highly specific compound and show that it preferentially targets the receptor on macrophages in blood vessels." He added that safety is a particular concern because "if you are treating atherosclerosis, you would have to take the drug for a long time-years, if not decades."

"The data look very promising, but the devil is in the details with pharmacological inhibitors. In order to take advantage of these data, they or someone else will need to get an inhibitor with a good therapeutic index worthy of preclinical development and medicinal chemistry," said Roger Sabbadini, founder, VP and CSO of Lpath Inc.

Lpath has two antibodies against sphingosine-1-phosphate: ASONEP and iSONEP. The former has completed Phase I testing for cancer and inflammation, and the latter has completed Phase I testing in age-related macular degeneration (AMD), diabetic retinopathy, glaucoma and other ocular disorders.

Takuwa told SciBX his lab "recently reported that S1PR2 is involved in the inhibition of tumor angiogenesis. Another recent paper showed that S1PR2 has some protective role in a mouse anaphylaxis model. Therefore, in relation to these, appropriate caution should be taken in clinical applications of an S1PR2 antagonist."
But, despite the concerns, Takuwa added that "in our mouse study, JTE-K1 did not show overt toxicity at the employed dose after threemonth administration."

\section{Plaque to the future}

In addition to safety concerns, finding an appropriate patient population for S1PR2 antagonists would be important. Indeed, Sabbadini noted that the JCI paper "does not show any resolution of atherosclerotic lesions, so this would need to be used as a preventative. The real questions are: Who are you going to treat if you develop the drug, and is there a large enough market for it?"

He added: "If you can find a group of high-risk patients, such as familial hypercholesterolemia patients who are at risk of serious cardiovascular events, it might be possible that the benefit outweighs the risk. People who have already had a cardiovascular event might also benefit because they are at high risk to have a second event within a few years."

Takuwa told SciBX that the findings in the JCI paper have been patented and the IP is available for licensing.

Martz, L. SciBX 3(43); doi:10.1038/scibx.2010.1285

Published online Nov. 4, 2010

\section{REFERENCES}

1. Wang, F. et al. J. Clin. Invest.; published online Oct. 18, 2010; doi:10.1172/JCl42315

Contact: Yoh Takuwa, Kanazawa University, Kanazawa, Japan e-mail: ytakuwa@med.kanazawa-u.ac.jp

2. Libby, P. Nature 420, 868-874 (2002)

3. Keul, P. et al. Arterioscler. Thromb. Vasc. Biol. 27, 607-613 (2007)

4. Skoura, A. et al. Arterioscler. Thromb. Vasc. Biol.; published online Oct. 14, 2010; doi:10.1161/ATVBAHA.110.213496

\section{COMPANIES AND INSTITUTIONS MENTIONED}

Charité-University Hospital Berlin, Berlin, Germany Japan Tobacco Inc. (Tokyo:2914; Osaka:2914), Tokyo, Japan Kanazawa University Graduate School of Medical Science, Kanazawa, Japan

Kanazawa University School of Medicine, Kanazawa, Japan Lpath Inc. (OTCBB:LPTN), San Diego, Calif. Novartis AG (NYSE:NVS; SIX:NOVN), Basel, Switzerland Teikyo University School of Medicine, Tokyo, Japan Tokyo University of Agriculture \& Technology, Fuchu, Japan The University of Tokyo Graduate School of Medicine, Tokyo, Japan Weill Cornell Medical College, New York, N.Y. 